\title{
Improvements on the quasifree absorption model for electron scattering
}

\author{
F. Blanco* \\ Departamento de Física Atómica Molecular y Nuclear, Facultad de Ciencias Físicas, Universidad Complutense, Avda. Complutense s/n, \\ E-28040 Madrid, Spain \\ G. García \\ Instituto de Matemáticas y Física Fundamental, Consejo Superior de Investigaciones Científicas, Serrano 121, 28006 Madrid, Spain
}

(Received 31 May 2002; published 6 February 2003)

\begin{abstract}
The quasifree nonempirical model proposed by Staszewka et al. in 1983 for the imaginary part of the electron scattering optical potential is revised, in order to improve its foundations, accuracy, and range of applicability. The importance of relativistic and many-body effects for heavy atoms is shown and some corrections proposed. A criterion for selecting the $\Delta$ parameter is discussed. The model is also shown to admit considerable simplifications without loss of accuracy. Calculated elastic (differential and integral) and inelastic cross sections for $\mathrm{He}$ to Xe noble gases and also for $\mathrm{N}_{2}, \mathrm{CO}$, and $\mathrm{CO}_{2}$ molecules are compared to experimental values.
\end{abstract}

DOI: 10.1103/PhysRevA.67.022701

PACS number(s): 34.10.+x, 34.80.-i, 31.15.-p

\section{INTRODUCTION}

Accurate data on cross sections for electron scattering by atoms and molecules are currently recognized as being very valuable due to their application in several areas of technological interest (modeling and diagnostics of plasma processes, Monte Carlo simulation of the interaction with matter, or radiation detector development) and environmental applications $\left(\mathrm{O}_{2}, \mathrm{O}_{3}, \mathrm{CCl}_{4}\right.$, and $\mathrm{CF}_{4}$ molecules $)$. This work is a consequence of several previous ones [1-4] devoted to combine experimental results with theoretical calculations, obtaining elastic and inelastic integral cross sections for electron scattering by molecules and noble gases in a wide energy range. As a consequence we have realized the importance of approximate $a b$ initio procedures of reasonable accuracy for evaluating inelastic processes.

As is well known, calculations ignoring inelastic processes do not allow the determination of total cross sections but provide good (somewhat overestimated) differential and integrated elastic values. In these cases, a complete description of the collision is not obtained and absorption contributions must be estimated separately [1-4]. Unfortunately, most available $a b$ initio treatments including inelastic contributions have a restricted range of applicability or become complicated when applied to situations of practical interest. Consequently, most treatments for complex atoms or molecules are of a semiempirical nature, being of limited confidence in the absence of experimental data.

We will consider here an approximate $a b$ initio model known as the quasifree absorption model. There are numerous calculations based on this model [5-13] but, due to some deficiencies of the original formulation, most authors use semiempirical variants for it. Nevertheless, it has been shown $[14,15]$ that, when properly amended, this model results in a useful treatment of reasonable accuracy and sim-

*Email address: pacobr@fis.ucm.es plicity, while maintaining its $a b$ initio nature and wide range of applicability.

This work is a continuation of $[14,15]$, devoted to improving the quasifree model proposed by Staszewka et al. [16] based on the suggestion of Goldberger [17] for an analogous situation in nuclear physics. In this model, the inelastic interaction of the incident electron with the target (atom or molecule) arises from its dispersion by the target electrons. While the two-body $e^{-}-e^{-}$elastic collisions are calculated as if the target electrons were free, target properties enter as boundary conditions for the binary collisions, spatial and momentum density distributions, and Pauli-principle restrictions on the allowed final states for colliding electrons. Since its introduction, the model has been applied to many atoms and molecules [5-9], but to our knowledge Refs. [14,15] are the only attempts to improve the model on a nonempirical basis.

As the fundamentals of the model have been extensively described elsewhere $[14,15,16,18]$, only a brief summary will be given here: Taking into account a local imaginary potential $i V^{a}$ corresponds to an $-2 V^{a}$ absorption per unit time [19], and assuming $u(\mathbf{r}, E)=\sqrt{2[E-V(r)]}$ as the velocity corresponding to the local kinetic energy $E-V(\mathbf{r})$ of the scattering electron, $V^{a}(r, E)=-\frac{1}{2} \rho(r) u \bar{\sigma}(r, E)$. Obtaining the explicit form is reduced to calculating [14,16-18] the effective collision cross section $\bar{\sigma}$ for an electron traversing a $\rho(\mathbf{r})$ electron charge density for which a Fermi velocity distribution is assumed.

The main contribution of Ref. [14], apart from an errata correction, was an improved treatment of the binary $e^{-}-e^{-}$ interactions, showing that the corresponding Mott treatment very approximately results in two terms for the final potential $V^{a}=V_{R}^{a}+V_{C}^{a}$. The first term $V_{R}^{a}$ is the one that would arise assuming the simple Rutherford expression for the $e^{-}-e^{-}$ binary interaction, while the correcting $V_{C}^{a}$ term is negligible in most cases.

The main contributions of Ref. [15] were a symmetrical treatment of the interacting electrons (originally considered 
as distinguishable) and a tentative correction for many-body effects.

Here we will propose some additional modifications based on ab initio arguments, and discuss their effect on the numerical results. A definitive solution to the problem is not claimed, but the resulting model is more accurate and of wider applicability.

Section II A will discuss how to remove an undesirable characteristic of the present model. In Sec. II B, we will describe some modifications necessary for the application to heavier atoms. Section II C proposes a criterion for choosing the $\Delta$ parameter in order to include some contribution from nonionizing processes. Section II D presents a simplified version of the model that can make it easier to use and interpret without losing accuracy, by means of identifying and dropping nonsignificant terms. The final two sections describe some numerical results for light to heavy atoms ( $\mathrm{He}$ to $\mathrm{Xe}$ in Sec. III A) and three molecules $\left(\mathrm{N}_{2}, \mathrm{CO}\right.$, and $\mathrm{CO}_{2}$ in Sec. III B).

In all calculations, atomic units will be used: Energy in hartrees $(27.212 \mathrm{eV})$, length in Bohr radius, $h / 2 \pi=m=e$ $=1$.

\section{THEORY}

\section{A. Restoring the local velocity in the model}

As indicated, the explicit expression for the model absorption potential $V^{a}(r, E)$ in the quasifree approximation results from the evaluation of the effective collision cross section $\bar{\sigma}$ for an electron traversing an electron cloud, for which a Fermi velocity distribution is assumed. This means considering inside the atom the collision of the incident electron of local velocity $\mathbf{u}$ with a target electron of local velocity $\mathbf{k}$, and then integrating the corresponding $d \sigma / d \Omega$ for all the allowed final states, and averaging over all the $\mathbf{k}$ Fermi distributed values. As a consequence, the final expression $\bar{\sigma}(r, E)$ explicitly depends [17] on the value of the local velocity $u=\sqrt{2(E-V)}$. Nevertheless, up to now, in all versions of the model $[14,15,16,18]$ this local velocity $\mathbf{u}$ has been replaced by the incident velocity $p=\sqrt{2 E}$. As has been shown [14], the use of the desirable value $u$ resulted in absurdly large values of the total inelastic cross section at low incident energies.

In order to understand this situation, it must be noted that, after accelerating in the atomic potential $V$, low-energy incident electrons can gain very large energies and then, satisfying the Pauli restrictions of the model, they are allowed to remove even very tightly bound internal atomic electrons. While such a process can be allowed "from the point of view" of the interacting electrons, it clearly means an energy-conservation violation for the whole electron-atom system. This situation can be easily remedied by incorporating into the model an energy-conservation boundary condition (similar to the two other Pauli blocking conditions of the model).

The energy-conservation restriction incorporated is the obvious one: If the kinetic energy of the incident electron is lower than the binding energy of the target electron, the inelastic interaction is not allowed. An explicit formulation of
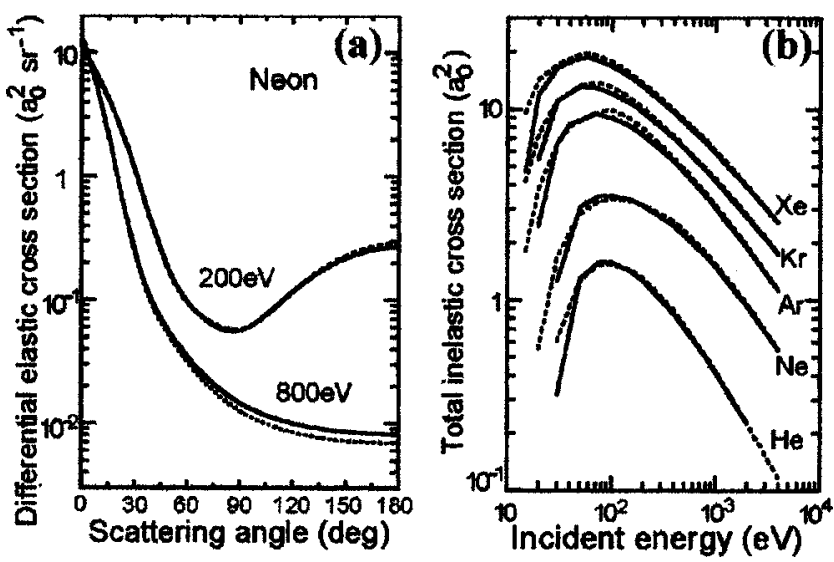

FIG. 1. Effect of the primitive $u \rightarrow p$ procedure. Dotted line, old version replacing local velocity $u$ by incident velocity $p$. Solid line, new version with the right $u$ value and an energy conservation restriction. (a) A sample of differential elastic cross sections for neon; (b) total inelastic cross section for He to Xe.

this restriction requires some estimation of the "local binding energy" for target electrons. While for weakly bound external electrons this can be the $\Delta$ threshold energy of the model, for internal electrons a good estimate could be the binding potential (the average potential seen by each atomic electron arising from the nucleus plus the remaining $Z-1$ electrons).

Therefore, our proposal will be using the right $u$ value (instead of $p$ ) in all the $V^{a}$ expressions, and the inclusion of the new factor $H\left(E_{\mathrm{in}}-\Delta+V_{b}\right)$, where $H$ is the usual Heaviside step function and $V_{b}(r)=V(r)(Z-1) / Z-1 / r$ is an estimation of the potential due to $Z-1$ electrons plus the nucleus.

Figure 1 compares both versions of the model for total inelastic cross sections in $\mathrm{He}$ to $\mathrm{Xe}$, and for some differential elastic ones in neon. The details of the numerical calculations are given below in Sec. III. It can be noted that although the expressions involved in both versions are quite different, the modification for the numerical results is small. That means the old $u \rightarrow p$ procedure, unless somewhat arbitrary, was not a bad approximation.

\section{B. Relativistic and many-body corrections}

We will discuss here the convenience of including relativistic and many-body effects for heavy atoms ( $\mathrm{Kr}$ and larger). The convenience of including relativistic corrections in the Schrödinger equation for the incident electron does not arise from the range of incident energies that will be considered here (up to a few $\mathrm{keV}$ ). Relativistic effects arise from the larger energies an incident electron acquires inside a heavy atom, where very strong potentials are present. The details of the adopted treatment will be commented on in Sec. III.

The numerical results for He to Xe noble gases show that relativistic corrections have no effect on the calculated total cross sections. Only $\mathrm{Kr}$ and $\mathrm{Xe}$ differential cross sections are affected for large angles (and in the case of $\mathrm{Kr}$ only at 200 $\mathrm{eV}$ or lower energies). Figure 2 compares the results for $\mathrm{Xe}$ at $250 \mathrm{eV}$ with and without relativistic corrections in the 


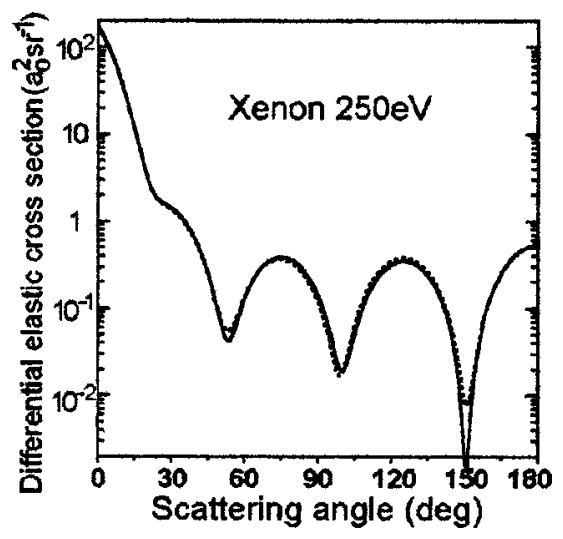

FIG. 2. Comparison for xenon of the results with (full line) and without (dotted line) inclusion of relativistic corrections for the incident electron.

incident electron. By omitting the absorption potential, a similar plot results, but then our relativistic-corrected curve is quite close to the pure elastic relativistic values given by Fink et al. [20].

Contrary to the small relativistic corrections, many-body effects, not taken into account by the original model, are very important even for a relatively small atom like $\mathrm{Ne}$, as shown in our previous work [14]. Clearly a precise description of heavier atoms would require a detailed treatment of manybody effects. Since such a treatment is not available now, we decided to introduce the necessary corrections at least in an approximate way, as follows.

In our previous work we introduced the notion of screening of the inner electrons by the outer ones, and consequently corrected the absorption potential by a factor $C_{\mathrm{scr}}(r)$ $=e^{\lambda_{0}(r)}$, where $\lambda_{0}(r)=\int_{r}^{\infty} 2 V^{a}(t) / u(t) d t<0$ is the "optical depth" corresponding to one-dimensional situations or to head-on incidence [Fig. 3(a)]. Taking into account that much larger paths are actually involved inside the atom [Fig. 3(b)], the above correction is clearly underestimated. The mean value $\left\langle\lambda_{\theta}(r)\right\rangle_{\Omega}$ obtained by averaging over all $r$ orientations [Fig. 3(c)] would be much more realistic, but it results in a double integral of annoying numerical implementation. After checking that the simpler expression $\lambda(r)$ $=\int_{r}^{\infty}\left[2 V^{a}(t) / u(t)\right]\left[t / \sqrt{t^{2}-r^{2}}\right] d t$ corresponding to transver-
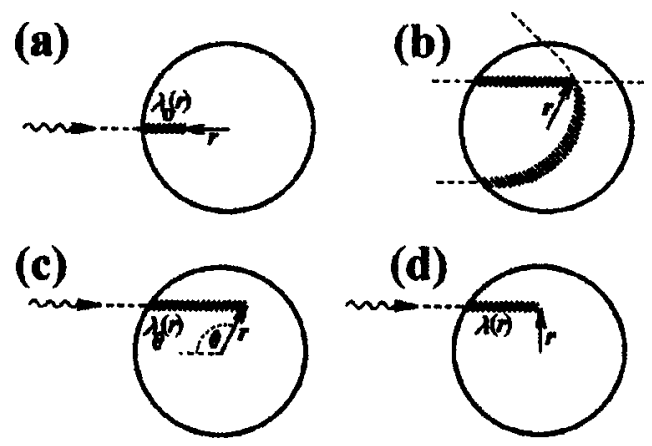

FIG. 3. Illustrative of the classical paths considered for the approximate screening correction. (a) Old version, (b) other paths involved, (c) averaging on straight paths, (d) adopted correction. sal incidence [Fig. 3d] gives much the same numerical results as $\left\langle\lambda_{\theta}(r)\right\rangle_{\Omega}$, we decided to adopt it.

Another questionable assumption of the original model, also related to many-body processes, is considering the probability of interacting with $Z$ electrons as $Z$ times the probability of interacting with one electron: if inside a region of size $\lambda^{3}(\lambda=2 \pi / u$ the local wavelength of the incident electron) more than one target electron were present, it would not be physically reasonable to consider interaction with each of them as independent of the others (even if target electrons are considered to behave as independent of each other). By denoting as $\rho_{1}(r)=\rho(r) / Z$ the probability distribution of one of the target electrons, the probability for that electron to be inside a $\lambda^{3}$ region is $\rho_{1} \lambda^{3}$, and the probability for "at least one electron" to be inside the $\lambda^{3}$ region is not $Z \rho_{1} \lambda^{3}$ $=\rho \lambda^{3}$, but $1-\left(1-\rho_{1} \lambda^{3}\right)^{Z} \approx 1-e^{-Z \rho_{1} \lambda^{3}}=1-e^{-\rho \lambda^{3}}$. This probability can be written as $\rho_{\text {ef }} \lambda^{3}$ by denoting $\rho_{\text {ef }}$ $=1 / \lambda^{3} /\left(1-e^{-\rho \lambda^{3}}\right)$ or better $\rho_{\mathrm{ef}}=\rho_{\mathrm{sat}}\left(1-e^{\left.-\rho / \rho_{\mathrm{sat}}\right)}\right.$, where $\rho_{\text {sat }}=1 / \lambda^{3}=u^{3} /(2 \pi)^{3}$ can be interpreted as the maximum target electron density an incident electron with local wavelength $\lambda$ can resolve. Thus for the case of multielectron atoms, we propose using $V^{a}(r, E)=-\frac{1}{2} \rho_{\mathrm{ef}}(r) u \bar{\sigma}(r, E)$ instead of the former $V^{a}(r, E)=-\frac{1}{2} Z \rho_{1}(r) u \bar{\sigma}(r, E)$. The difference becomes significant only in the regions with very large charge density.

It must be noted that applying the above two approximate corrections does not mean correcting twice for the same effect: while the first is related to the probability for the incident electron to reach the position $\mathbf{r}$, the second is related to the probability of interacting with a target electron at $\mathbf{r}$ once arriving there. Both corrections deal with complementary manifestations of the many-body situation. In spite of the heuristical nature of the above reasoning, we consider that the resulting approximate corrections go in the right direction, as confirmed by the numerical results, and so they will be maintained until a sound treatment becomes available.

Figure 4 compares the old and the new correction procedures with the uncorrected results. For the differential elastic cross sections, the results are quite different (the new correction resulting in an appreciable improvement when compared to available experimental data). For total inelastic cross sections, the effect of the old correction procedure was small, while the new one resulted in a stronger reduction at the maximum.

\section{Determination of the $\Delta$ parameter}

It must be remembered that the effective cross section $\bar{\sigma}$ arises from integration $[14,16,17]$ of the binary electronelectron differential one $d \sigma_{b} / d \Omega$ over all the continuum final states $\left(\mathbf{p}^{\prime}, \mathbf{k}^{\prime}\right)$ energetically allowed for the scattered electrons $\left(p^{\prime 2} / 2>k_{F}^{2} / 2+\Delta\right.$ and $\left.k^{\prime 2} / 2>k_{F}^{2} / 2+\Delta\right)$. This means the $\Delta$ parameter represents the threshold energy for continuum states: only ionization processes are taken into account, excitation to discrete levels being ignored by the model.

Using for $\Delta$ any value under the ionization potential can be understood as a desirable attempt to include in the model some contribution from excitation to discrete bound states, 

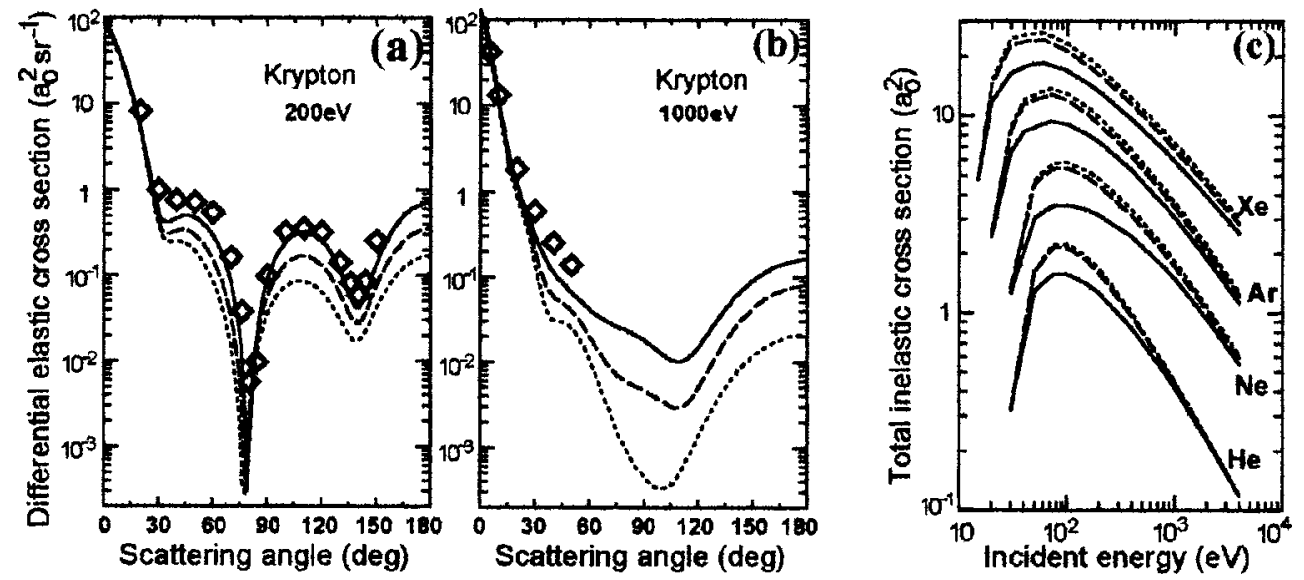

FIG. 4. Comparison of the many-body correction procedures: - - proposed corrections; - - - old version; $\cdots$, uncorrected results. (a), (b) Samples for differential elastic cross section in $\mathrm{Kr}$; (c) total inelastic cross section for $\mathrm{He}, \mathrm{Ne}, \mathrm{Ar}$, and $\mathrm{Xe}$.

but requires some discussion for the appropriate value: The original proposal for $\Delta[16]$ was the energy gap between the ground state and the first discrete level. Unfortunately, this value is unacceptable for most atoms because it is so small (for example, $0.002 \mathrm{eV}$ in carbon) that nonsense $\sigma$ values would result.

Probably the most judicious choice for the $\Delta$ parameter should require a careful discussion for each particular system. Nevertheless, taking into account that the contribution of optically forbidden transitions to the excitation cross section is usually very small compared to the contribution of those optically allowed, we consider a reasonable general assumption could be taking $\Delta$ as the gap between the ground state and the first level optically allowed from the ground one (first resonant level).

Figure 5 illustrates the inelastic cross sections resulting when using for $\Delta$ the ionization potential (ignoring excitation processes) and the larger ones resulting when correcting for them (proposed value of $\Delta$ ).

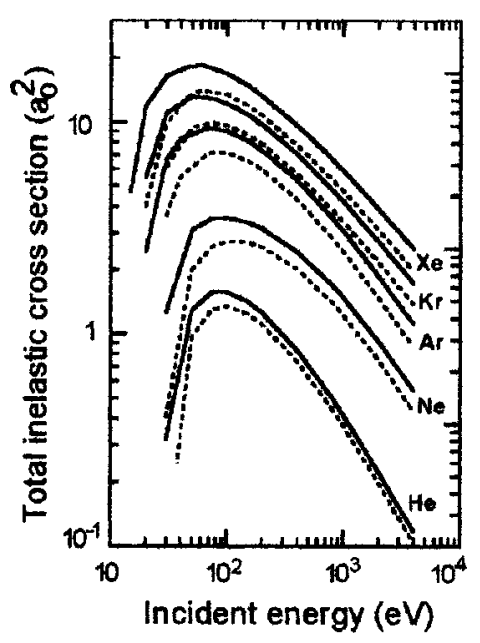

FIG. 5. Total inelastic cross sections for He to Xe. Dotted line is the result when using for $\Delta$ the ionization potential, ignoring excitation processes. Full lines are the results when using for $\Delta$ the energy gap between the ground and first resonant level (correcting for excitation processes).

\section{Resulting expressions and usable simplifications}

\section{Summary of expressions for the proposed form of the model}

In order to summarize the above discussions, we present here the final form proposed for the quasifree absorption model potential after inclusion of all the proposed modifications:

$$
\begin{gathered}
V_{\mathrm{cor}}^{a}(r)=V^{a}(r) C_{\mathrm{scr}}(r), \\
C_{\mathrm{scr}}(r)=\exp \left(\int_{r}^{\infty} \frac{2 V^{a}(t)}{u(t)} \frac{t d t}{\sqrt{t^{2}-r^{2}}}\right) \\
V^{a}(r)=V_{R}^{a}(r)+V_{C}^{a}(r), \\
V_{R}^{a}=\frac{-2 \pi \rho_{\mathrm{ef}}}{u} H\left[E_{\mathrm{in}}+V_{\mathrm{b}}(r)-\Delta\right] \\
\times\left[\frac{1}{\Delta}-\frac{u^{2}-\Delta-\frac{3}{5} k_{F}^{2}}{\left(u^{2}-k_{F}^{2}-\Delta\right)^{2}}+H(\delta) \frac{2 \delta^{5 / 2} u^{5}}{5 k_{F}^{3}\left(u^{2}-k_{F}^{2}-\Delta\right)^{2}}\right] \\
V_{C}^{a}=\frac{2}{\pi} H\left[E_{\mathrm{in}}+V_{\mathrm{b}}(r)-\Delta\right]\left\{f_{\delta}\left(k_{F} / u\right)-f_{\delta}\left[H(\delta) \delta^{1 / 2}\right]\right\}
\end{gathered}
$$

Here $E_{\text {in }}$ stands for the incident kinetic energy, $u\left(r, E_{\text {in }}\right)$ $=\sqrt{2\left[E_{\mathrm{in}}-V(r)\right]}, \rho_{\mathrm{ef}}=\rho_{\mathrm{sat}}\left(1-e^{\left.-\rho / \rho_{\mathrm{sat}}\right)}, \rho_{\mathrm{sat}}=u^{3} /(2 \pi)^{3}, \delta\right.$ $=\left(2 k_{F}^{2}+2 \Delta-u^{2}\right) / u^{2}, k_{F}(\rho(\mathbf{r}))=\left[3 \pi^{2} \rho(\mathbf{r})\right]^{1 / 3}, \Delta$ is the energy gap between ground and first resonant states, $V_{b}(r)$ is estimated from the effective atomic potential $V(r)$ as $V_{b}(r)$ $=V(r)(Z-1) / Z-1 / r$, and the $f_{\delta}$ function [14] is

$$
f_{\delta}(x)=\int_{0}^{x} d t t^{2}\left(t^{2}-\delta\right) \int_{-1}^{1} \frac{d s}{\left(t^{2}+1-2 t s\right)^{2}\left(t^{2}+1+2 t s\right)^{1 / 2}}
$$

for which an approximate expression [14] is 


$$
\begin{aligned}
f_{\delta}(x) \approx & \frac{x(1-\delta)}{4(1-x)}+\frac{[11-x+(x-3) \delta] \ln (1-x)}{16} \\
& +\left(a_{1}-\delta b_{1}\right) x+\left(a_{2}-\delta b_{2}\right) x^{2}
\end{aligned}
$$

with $a_{1}=0.4353, a_{2}=0.01233, b_{1}=-0.1084$, and $b_{2}$ $=0.05691$.

\section{Alternative simplified expressions}

To our knowledge, all previous works based on the present model used the complete complicated expressions similar to those above. Nevertheless the above expressions contain several terms with a negligible contribution in most situations. Consequently simplified expressions can be used without appreciable loss of accuracy.

A numerical evaluation of the different terms in expressions (1a)-(1e) for He to Xe noble gases indicates that without appreciable loss of accuracy, the following holds true.

(a) The very small $V_{C}^{a}(r)$ and $H(\delta) \delta^{5 / 2}$ terms can be ignored.

(b) Compared to the $1 / \Delta$ term, the $\left(u^{2}-\Delta-\frac{3}{5} k_{F}^{2}\right) /\left(u^{2}\right.$ $\left.-k_{F}^{2}-\Delta\right)^{2}$ term is also small over most of the $r$ range, and so the small change $\frac{3}{5} k_{F}^{2} \rightarrow k_{F}^{2}$ simplifies it to $1 /\left(u^{2}-k_{F}^{2}\right.$ $-\Delta$ ) with no significant change in the calculations. Furthermore, approximating $u^{2}=2 E_{\text {in }}-2 V \approx 2 E_{\text {in }}+k_{F}^{2}$ (for a Thomas-Fermi neutral atom this would be exact), this term can be finally written as $1 /\left(2 E_{\text {in }}-\Delta\right)$.

(c) We have also found that for most calculations, the $C_{\mathrm{scr}}(r)=\exp \int_{r}^{\infty}\left[2 V^{a}(t) / u(t)\right]\left[t / \sqrt{t^{2}-r^{2}}\right] d t$ expression can be substituted without appreciable change in the results by $\widetilde{C}_{\mathrm{scr}}(r)=\exp 2 \int_{r}^{\infty} 2 V^{a}(t) / u(t) d t$, which is of easier numerical evaluation.

As a summary, a simplified version of the model can be proposed in the form

$$
\begin{gathered}
\widetilde{V}_{\mathrm{cor}}^{a}(r)=\widetilde{V}^{a}(r) \widetilde{C}_{\mathrm{scr}}(r), \\
\widetilde{C}_{\mathrm{scr}}(r)=e^{2_{r}^{\infty} 2 \widetilde{V}^{a}(t) / u(t) d t} \\
\widetilde{V}^{a}=\frac{-2 \pi \rho_{\mathrm{ef}}}{u} H\left[E_{\mathrm{in}}+V_{b}(r)-\Delta\right]\left[\frac{1}{\Delta}-\frac{1}{2 E_{\mathrm{in}}-\Delta}\right] .
\end{gathered}
$$

It must be noted that unless the simplicity of the above result could make it of interest for further applications, the above simplifications are of a purely numerical nature.

As Fig. 6 shows, the results from the complete and simplified versions of the model are very similar for the total inelastic cross section of He to Xe. Differential elastic values are not plotted as they are almost identical.

\section{CALCULATiONS}

\section{A. Numerical calculations for noble gas atoms}

In order to check the usefulness of the model and the relevance of some of the proposed modifications, we applied it to the He to Xe noble gases. The detailed procedure of the numerical calculations has been extensively described elsewhere $[1,2,3,6,14,15]$, and so only a brief summary follows.

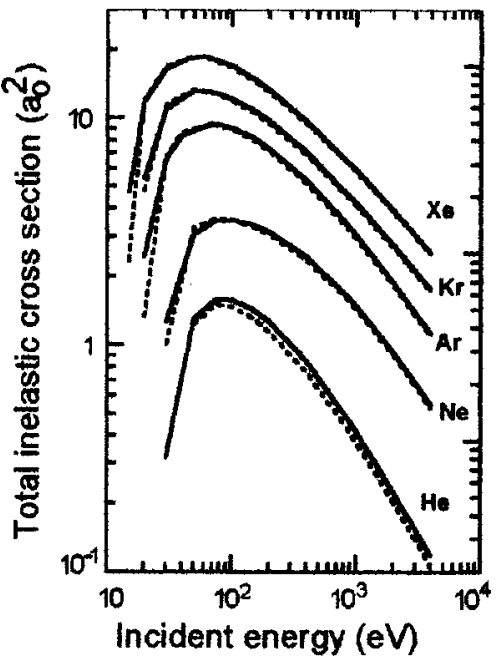

FIG. 6. Comparison for He to Xe of the total inelastic cross sections calculated from the complete (full line) and simplified (dotted line) versions of the model.

For our purposes, the electron-atom interaction is represented by the approximate optical potential

$$
V_{\mathrm{opt}}(r)=V(r)+i V^{a}(r)=V_{s}(r)+V_{e}(r)+V_{p}(r)+i V^{a}(r) .
$$

Here the imaginary part $V^{a}(r)$ was the absorption potential in complete form [Eqs. (1a)-(1e)], and the real part $V(r)$ is the effective atomic potential including three terms: $V_{s}(r)$ is the static potential calculated by using the charge density deduced from Hartree-Fock [21] atomic wave functions incluiding relativistic corrections. $V_{e}(r)$ is the exchange potential for which the semiclassical energy-dependent formula derived by Riley and Truhlar [22] is used. $V_{p}(r)$ represents the target polarization potential in the form given by Zhan et al. in Ref. [23].

The procedure adopted in considering relativistic effects for the incident electron involves the usual corrections [21] in the wave equation, resulting in the use of an effective $g_{l}$ wave function and potential [20]. Thus, except for ignoring the difference between $\delta_{l}$ and $\delta_{-l-1}$, our treatment follows the one given by Fink et al. [20]. As mentioned above, relativistic corrections for the incident electron are only of small importance here. Nevertheless, they have been included in all our calculations, as once they are implemented in the numerical procedures they result in no extra work.

In order to obtain the $l$ th complex partial wave phase shift $\delta_{l}=\lambda_{l}+i \mu_{l}$, the scattering equation for the $u_{l}(r)$ radial wave functions has been numerically integrated [1] by means of an adaptive-step-size fourth-order Runge-Kutta algorithm [24] based on the variable-phase technique [25]. Once the corresponding $\delta_{l}$ phase shifts are obtained for the above potential, the elastic differential $d \sigma_{\mathrm{el}} / d \Omega$ and total absorption $\sigma_{\text {tot }}(E)$ cross sections result from their well-known partial wave and optical theorem expressions,

$$
f(\theta)=\frac{1}{2 i k} \sum_{l=0}^{l_{\max }}(2 l+1)\left(e^{\left.2 i \delta_{l}-1\right)} P_{l}(\cos \theta),\right.
$$




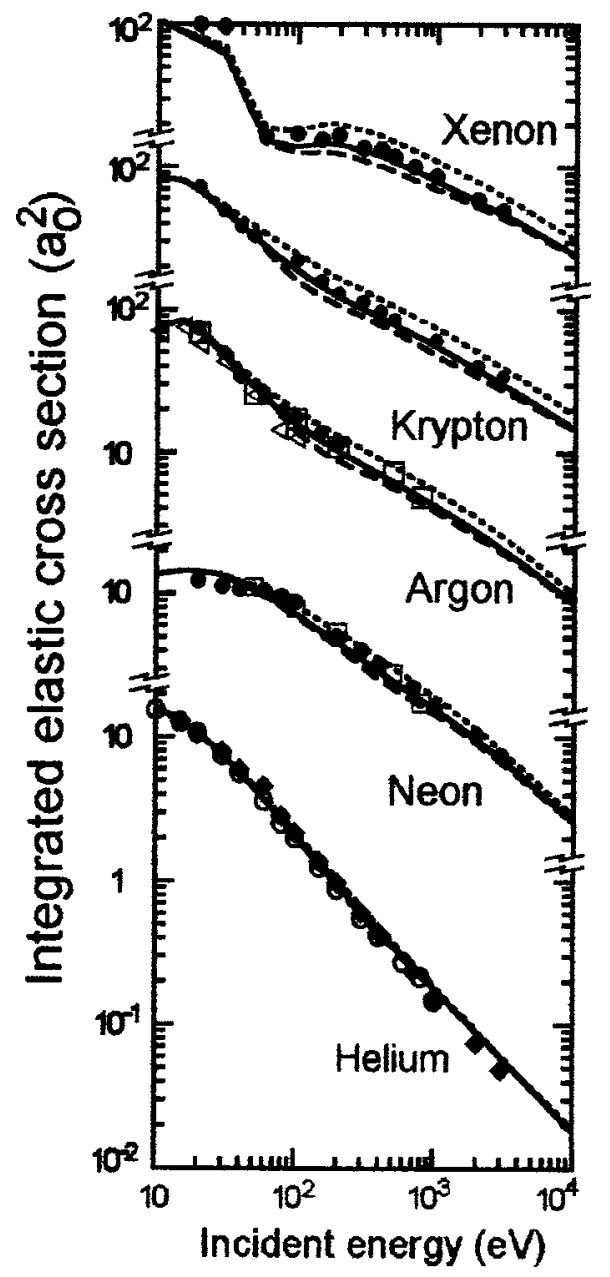

FIG. 7. Comparison for $\mathrm{He}$ to $\mathrm{Xe}$ of the calculated integral elastic cross section (curves) with some available experimental data (symbols): solid line is from the present model; dashed line (almost overlapping) is from the previous [15] model; dotted line is the pure elastic calculation omitting the imaginary absorption potential; $\bullet$, Ref. [26]; ๑, Ref. [27]; $\square$, Ref. [28]; ○, Ref. [29]; $\triangleleft$, Ref. [30]. Note the discontinuities in the axis.

$$
\frac{d \sigma_{\mathrm{el}}}{d \Omega}=|f(\theta)|^{2}, \quad \sigma_{\mathrm{tot}}(E)=\frac{4 \pi}{k} \operatorname{Im}\left(f_{\theta=0}\right) .
$$

Calculations were carried out both with the $i V^{a}(r)$ absorption term and without it (pure elastic). Figures 7-9 compare all the results with available experimental data. The results from the previous [15] model are also shown for comparison.

Figure 7 presents the integrated elastic cross sections, and Fig. 8 the total inelastic cross sections $\sigma_{\text {tot }}(E)-\sigma_{\text {elast }}(E)$. In all cases, the values from the modified model are similar to those from the previous one, and so also is the agreement with available experimental data.

Differential elastic cross sections are presented in Fig. 9 for low, medium, and large energies for which experimental data are available. The new results are usually larger for large angles, resulting in better agreement with available experimental data. This is especially remarkable for medium to large energies, where new results a factor $\times 2$ to $\times 10$ larger

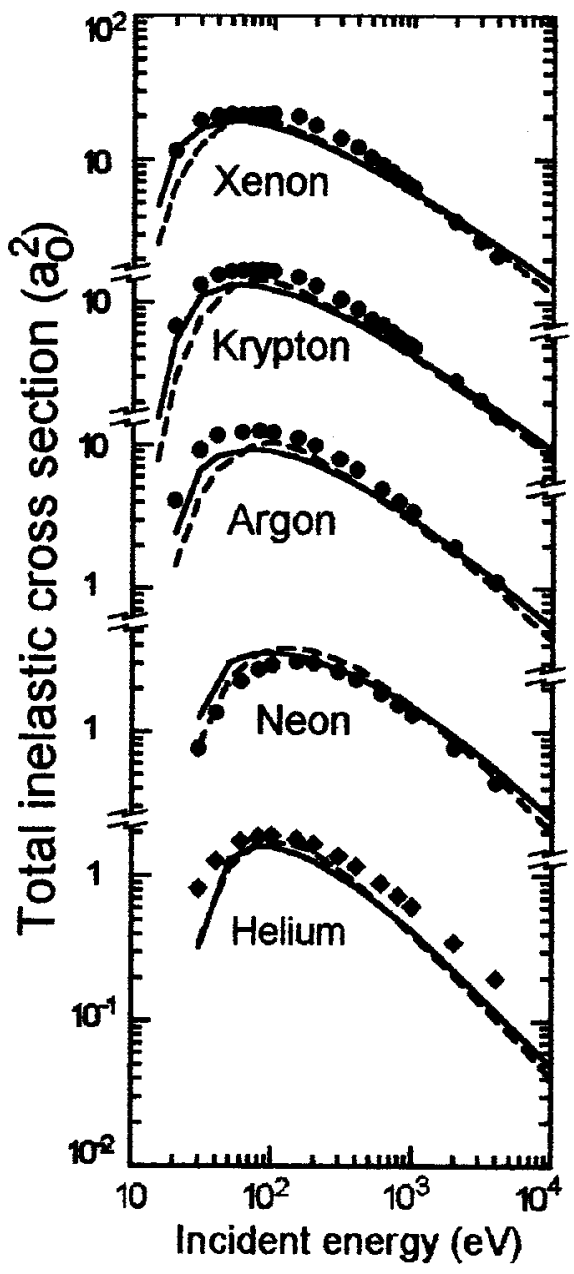

FIG. 8. Comparison for He to Xe of the calculated total inelastic cross section. All symbols and line styles are the same as Fig. 7.

towards experimental data are obtained. For integrated elastic cross sections, Fig. 7 also indicates that in all cases the new results are closer to the experimental ones.

It is very interesting to compare the results from calculations including the $i V^{a}(r)$ term with those obtained when ignoring it (pure elastic): The former provides us with good total, absorption, integrated elastic, and differential elastic values (these last are somewhat underestimated at large energies). The second one does not allow determination of total or absorption cross sections and somewhat overestimates differential elastic values. Thus (only for determination of differential elastic values) both calculations can be considered as complementary for the time being, as they result in useful upper and lower bounds for the experimental ones.

\section{B. Numerical calculations for some molecules}

In order to illustrate the possibilities of the model for calculation of molecular cross sections, we will present here some results for $\mathrm{N}_{2}, \mathrm{CO}$, and $\mathrm{CO}_{2}$ cases. Our molecule calculations will be based here on the independent atom model (IAM) $[6,8,19,38,39]$ and the usual expression [19] for multicenter dispersion, 

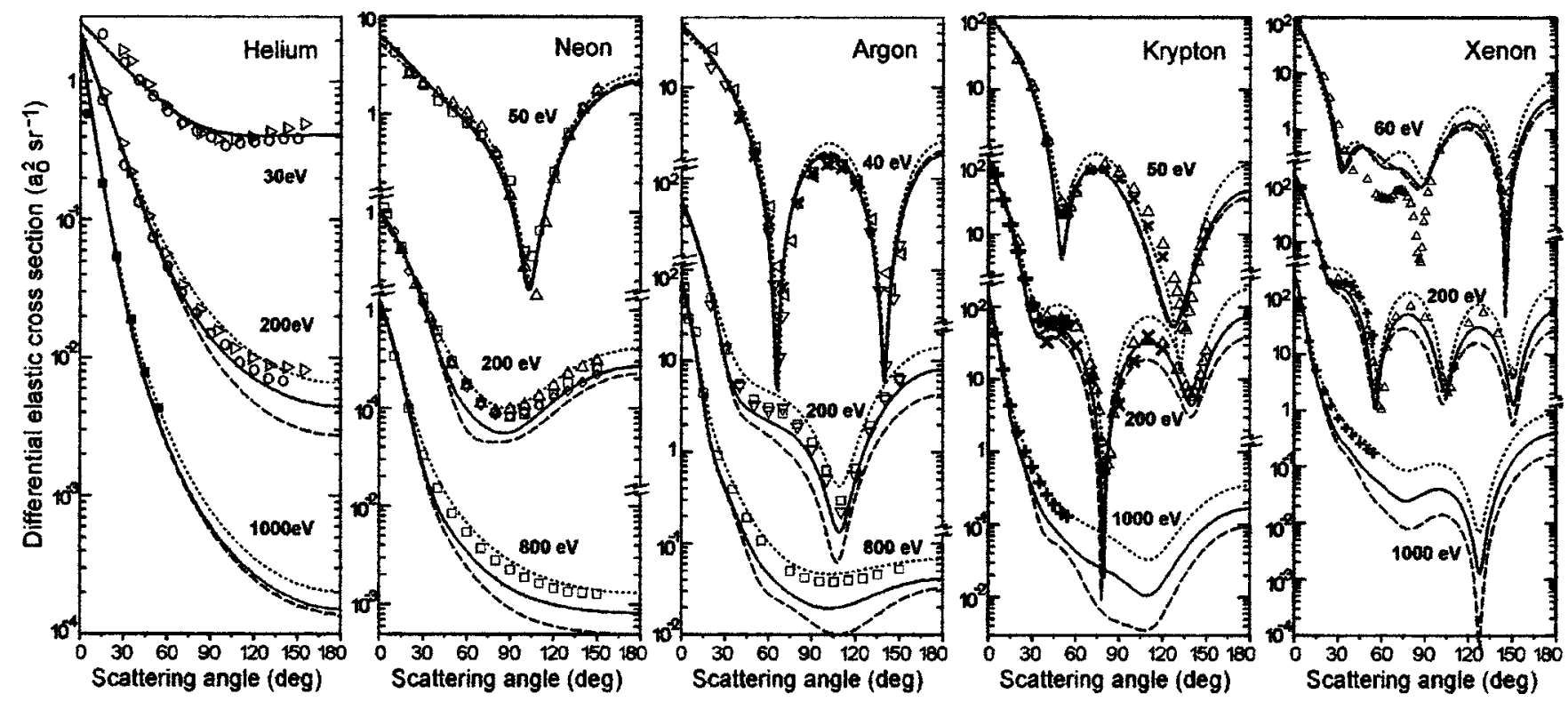

FIG. 9. Differential cross sections for He to Xe noble gases. All symbols and line styles same as Fig. 7 and also $\boldsymbol{\square}$, Ref. [31]; $\triangleright$, Ref. [32]; $\triangle$; Ref. [33]; $\nabla$, Ref. [34]; $\diamond$, Ref. [35]; $\times$, Ref. [36]; + , Ref. [37]. Note the discontinuities in the axis.

$$
F(\theta)=\sum_{\text {atoms }} f_{i}(\theta) e^{i \mathbf{q} \cdot \mathbf{r}_{i}}
$$

where $\mathbf{q}=\mathbf{K}_{\text {out }}-\mathbf{K}_{\text {in }}$ is the momentum transfer and $f_{i}(\theta)$ are the atomic dispersion functions. According to the optical theorem, the resulting total cross section is then

$$
\begin{aligned}
\sigma_{\text {total }}^{\text {molecule }} & =\frac{4 \pi}{k} \operatorname{Im} F(\theta=0)=\frac{4 \pi}{k} \sum_{\text {atoms }} \operatorname{Im} f_{i}(\theta=0) \\
& =\sum_{\text {atoms }} \sigma_{\text {total }}^{\text {atom }},
\end{aligned}
$$

a result known $[6(\mathrm{a})]$ as the additivity rule. After averaging the differential cross section $|F(\theta)|^{2}$ over all molecule orientations [[6b], [19]], one also obtains

$$
\frac{d \sigma^{\text {molec }}}{d \Omega}=\sum_{i, j} f_{i}(\theta) f_{j}^{*}(\theta) \frac{\sin q r_{i j}}{q r_{i j}},
$$

where $q=2 k \sin \theta / 2, r_{i j}$ is the distance between $i$ and $j$ atoms, and $\sin q r_{i i} / q r_{i i}=1$. Unfortunately Eq. (7) is not appropriate for calculation of integrated elastic values due to its poor normalization [this is very clear for the pure elastic dispersion, where $F(\theta)$ violates the optical theorem $\int_{4 \pi}|F(\theta)|^{2} d \Omega \neq(4 \pi / k) \operatorname{Im} F(\theta=0)$, even in the simplest case of two identical atoms]. Taking into account that $\sin q r_{i j} / q r_{i j}$ factors are usually very small except for $i=j$, where they equal 1 , the above limitation can be remedied by approximating them as $\delta_{i j}$ while evaluating integral elastic values. This results again in an additivity rule relation,

$$
\sigma_{\text {integral elastic }}^{\text {molecule }}=\sum_{\text {atoms }} \sigma_{\text {integral elastic }}^{\text {atom }}
$$

The calculated values from Eqs. (6), (7), and (8) for $\mathrm{N}_{2}$, $\mathrm{CO}$, and $\mathrm{CO}_{2}$ molecules are compared in Figs. 10, 11, and 12 with available experimental or theoretical data.

As can be seen in the figures, the overall agreement between experimental and calculated values is good for differential cross sections. As with the noble gases case, the calculated values for medium to large angles tend to be lower than the experimental ones as energy increases. As for noble gases, the results from the present model are closer to the experimental data than the ones from the previous model, although those are not plotted here in order to simplify the figures. Values resulting both when including the $i V^{a}(r)$ absorption term and when omitting it (pure elastic) are shown for comparison, and, as for the atoms, they behave as upper and lower bounds for the experimental data.
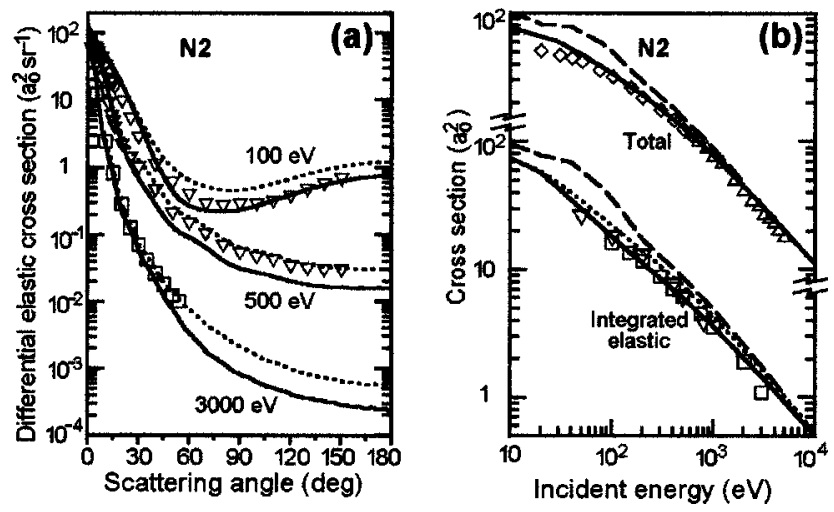

FIG. 10. Comparison for $\mathrm{N}_{2}$ of the calculated (curves) differential elastic (a) and total (b) cross sections, with some available experimental data (symbols): solid line is from the present model; dotted line is the pure elastic calculation omitting the imaginary absorption potential; dashed line is from Ref. [10]; $\square$, Ref. [31]; $\triangle$, Ref. [40]; $\diamond$, Ref. [41]; $\nabla$, Ref. [28]. Note the discontinuities in the axis. 

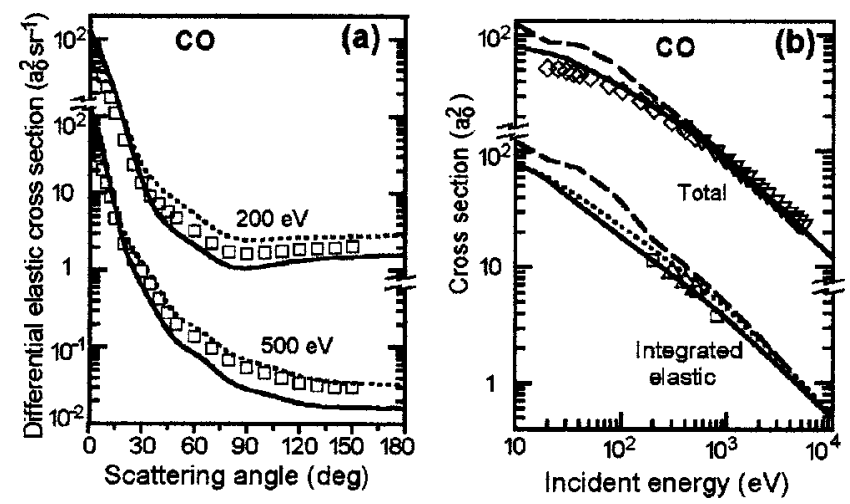

FIG. 11. Same as Fig. 10 but for the CO molecule, and $\square$, Ref. [28]; $\triangle$, Ref. [42]; $\diamond$, Ref. [43]; $\nabla$, Ref. [44]. Note the discontinuities in the axis.

The calculated total cross sections are in excellent agreement with experimental values at medium to large energies (100 eV and above). At lower energies, the agreement worsens, as expected from the low-energy failure of the IAM approximation. This is clearer for the largest $\mathrm{CO}_{2}$ molecule.

\section{CONCLUSIONS}

From the theoretical point of view, the modifications introduced in the quasifree scattering model potential remove undesirable characteristics of former treatments and approximately correct for many-body effects and nonionization processes. From the applied point of view, the model remains of an $a b$ initio nature and the new version gives improved results for He to Xe noble gas differential cross sections. The
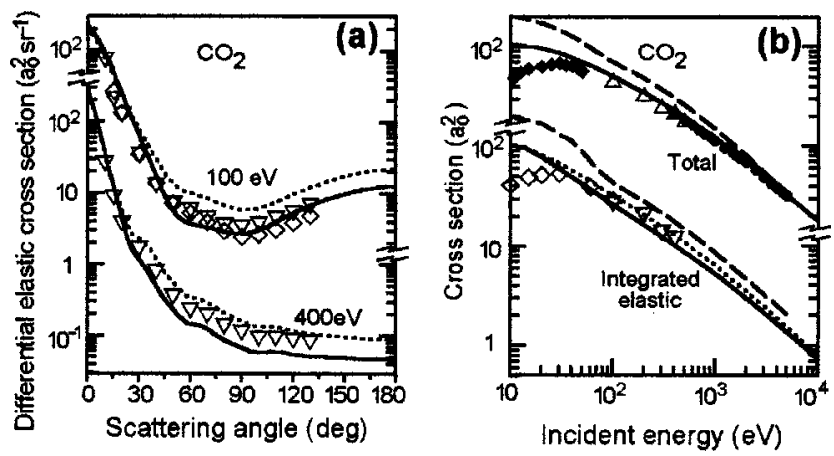

FIG. 12. Same as Fig. 10 but for the $\mathrm{CO}_{2}$ molecule, and $\diamond$, Ref. [41]; $\triangle$, Ref. [43]; $\diamond$, Ref. [45]; $\nabla$, Ref. [46]; $\bullet$, Ref. [47]. Note the discontinuities in the axis.

application to molecules is also satisfactory.

Thus we consider that the proposed form of the model results in a useful technique of reasonable accuracy and simplicity in studying electron scattering for a broad range of species.

Based on numerical considerations, a simplified version of the model is also discussed, resulting in much simpler expressions without appreciable loss of accuracy.

\section{ACKNOWLEDGMENTS}

This work was partially supported by the Spanish Programa Nacional de Promoción General del Conocimiento (Project BFM2000-0012) and Programa Nacional de Procesos y Productos Químicos (Project PPQ2000-0730-C02-01).
[1] G. García, M. Roteta, F. Manero, F. Blanco, and A. Williart, J. Phys. B 32, 1783 (1999).

[2] G. García, F. Blanco, and A. Williart, Chem. Phys. Lett. 335, 227 (2001).

[3] G. García and F. Blanco, Phys. Lett. A 279, 61 (2001).

[4] G. García and F. Blanco, Phys. Rev. A 62, 044702 (2000).

[5] A. Jain, B. Etemadi, and K. R. Karim, Phys. Scr. 41, 321 (1990).

[6] Y. Jiang, J. Sun, and L. Wan, (a) Phys. Rev. A 52, 398 (1995); (b) Phys. Lett. A 231, 231 (1997); (c) J. Phys. B 30, 5025 (1997).

[7] D. Raj and S. Tomar, J. Phys. B 30, 1989 (1997).

[8] S. P. Khare, D. Raj, and P. Sinha, J. Phys. B 27, 2569 (1994).

[9] K. N. Joshipura and P. M. Patel, Z. Phys. D: At., Mol. Clusters 29, 269 (1994).

[10] A. Jain and K. L. Baluja, Phys. Rev. A 45, 202 (1992).

[11] M. T. Lee, I. Iga, L. E. Machado, and L. M. Brescansin, Phys. Rev. A 62, 062710 (2000).

[12] I. Iga, M. T. Lee, M. G. P. Homem, L. E. Machado, and L. M. Brescansin, Phys. Rev. A 61, 022708 (2000).

[13] I. Iga, M. G. P. Homem, K. T. Mazon, and M. T. Lee, J. Phys. B 32, 4373 (1999).

[14] F. Blanco and G. García, Phys. Lett. A 255, 147 (1999).
[15] F. Blanco and G. Garcia, Phys. Lett. A 295, 178 (2002).

[16] G. Staszewska, D. W. Schwenke, D. Thirumalai, and D. G. Truhlar, Phys. Rev. A 28, 2740 (1983); J. Phys. B 16, L281 (1983).

[17] M. L. Goldberger, Phys. Rev. 74, 1269 (1948).

[18] G. Staszewska, D. W. Schwenke, and D. G. Truhlar, Phys. Rev. A 29, 3078 (1984).

[19] N. F. Mott and H. S. W. Massey, The Theory of Atomic Collisions, 3rd ed. (Clarendon Press, Oxford, 1971).

[20] D. Gregory and M. Fink, At. Data Nucl. Data Tables 14, 39 (1974); M. Fink and A. Yates, At. Data 1, 385 (1970).

[21] R. D. Cowan, The Theory of Atomic Structure and Spectra (University of California Press, London, 1981).

[22] M. E. Riley and D. G. Truhlar, J. Chem. Phys. 63, 2182 (1975).

[23] X. Z. Zhang, J. F. Sun, and Y. F. Liu, J. Phys. B 25, 1893 (1992).

[24] H. William, Numerical Recipes in Fortran, 2nd ed. (Cambridge University Press, Cambridge, 1994).

[25] F. Calogero, Variable Phase Approach to Potential Scattering (Academic, New York, 1967).

[26] F. J. de Heer and R. H. J. Jansen, J. Phys. B 10, 3741 (1977).

[27] F. J. de Heer, R. H. J. Jansen, and W. van der Kaay, J. Phys. B 12, 979 (1979). 
[28] R. D. DuBois and M. E. Rudd, J. Phys. B 9, 2657 (1976).

[29] D. F. Register, S. Trajmar, and S. K. Srivastava, Phys. Rev. A 21, 1134 (1980).

[30] R. Panajotovic, D. Filipovic, B. Marinkovic, V. Pejcev, M. Kurepa, and L. Vuskovic, J. Phys. B 30, 5877 (1997).

[31] R. H. J. Jansen, F. J. de Heer, H. J. Luyken, B. van Wingerden, and H. J. Blaauw, J. Phys. B 9, 185 (1976).

[32] T. W. Shyn, Phys. Rev. A 22, 916 (1980).

[33] J. F. Williams and A. Crowe, J. Phys. B 8, 2233 (1975).

[34] J. F. Williams and B. A. Willis, J. Phys. B 8, 1670 (1975).

[35] S. C. Gupta and J. A. Rees, J. Phys. B 8, 417 (1975).

[36] D. Cvejanovic and A. Crowe, J. Phys. B 30, 2873 (1997).

[37] R. H. J. Jansen and F. J. de Heer, J. Phys. B 9, 213 (1976).

[38] H. S. Massey, Electronic and Ionic Impact Phenomena (Clarendon, Oxford, 1969), Vol. 2.

[39] D. Raj, J. Phys. B 24, L431 (1991).

[40] G. Garcia, A. Perez, and J. Campos, Phys. Rev. A 38, 654
(1988).

[41] K. R. Hoffman, M. S. Dababneh, Y-F. Hseih, W. E. Kauppila, V. Pol, J. H. Smart, and T. S. Stein, Phys. Rev. A 25, 1393 (1982).

[42] J. P. Bromberg, J. Chem. Phys. 52, 1243 (1970).

[43] Ch. K. Kwan, Y-F. Heish, W. E. Kauppila, S. J. Smith, T. S. Stein, M. N. Uddin, and M. S. Dababneh, Phys. Rev. A 27, 1328 (1983).

[44] G. García, C. Aragón, and J. Campos, Phys. Rev. A 42, 4400 (1990).

[45] H. Tanaka, T. Ishikawa, T. Masai, T. Sagara, L. Boestaen, M. Takekawa, Y. Itikawa, and M. Kimura, Phys. Rev. A 57, 1798 (1998).

[46] I. Iga, M. G. P. Homem, K. T. Mazon, and M-T. Lee, J. Phys. B 32, 4373 (1999).

[47] G. García and F. Manero, Phys. Rev. A 53, 250 (1996). 